\title{
The LNQ25 and ELN PVT metrics exhibit a good sensitivity to sleep deprivation and are independent from the subject
}

LIÈGE

université
Philippe LATOUR and Marc VAN DROOGENBROECK

philippe. latour@ulg.ac.be, m. vandroogenbroeck@ulg.ac.be

Dpt. of Electricity, Electronics and Computer Science - University of Liège - Belgium

\section{Objectives}

We analyze and compare the sensitivity to sleep deprivation and the subject dependent variability of the PVT metrics performance, with a special emphasis on the time interval sizes.

\section{Introduction \& Context}

- Performance of people undergoing critical tasks (like driving) are impaired by the lowering of their vigilance level, due to sleep deprivation.

- Automatic systems compute an alertness level and detect drowsiness. Their assessment proceed by comparison with validated indices.

- We consider the 10min standardized PVT (Psychomotor Vigilance Test) as a validated index

\section{PVT metrics computed on short time intervals}

- Some metrics computed with all the reaction times (RT) of a $10 \mathrm{~min}$

PVT are good indicators of sleep deprived states.

- But, the assessment of "real-time" performances requires indices to be computed on much shorter time intervals (from $20 \mathrm{sec}$ to $2 \mathrm{~min}$ ).

Classification of PVT metrics into SDP or Non-SDP

- PVT metrics measure the performance of a person and we assume it to be related in some ways to the alertness level of this person.

- We separate the range of possible metrics values into two classes: Sleep Deprived state (SDP) and not in a Sleep Deprived state (Non-SDP).

\section{Materials \& Methods}

- 22 volunteers (11 males, 11 females, mean 22.2y., range 19-34 years)

- 28h sleep deprivation standard PVT protocol with six PVT sessions. [1]

- The first two PVT are in Non-SDP condition (9h30, $10 \mathrm{~h} 30$ Day 1 ) while the other PVT are in SDP condition (2h30, 3h30, 10h30, 11h30 Day 2).

- The subjects had a normal sleep-wake cycle during the week prior the experiment (no sleep deprivation, jet-lag or shift work and no medication).

\section{PVT metrics}

We compute the usual PVT metrics; meanRT, meanRS (Reaction Speed) and $\mathbf{L} \mathbf{N 5 0 0}$ (500ms lapses number). We also compute the LNQ25 (adaptive lapses number computed with a subject dependent threshold) and the ELN (Expected Lapse Number, computed from a subject dependent estimation of the lapse probability). [2]
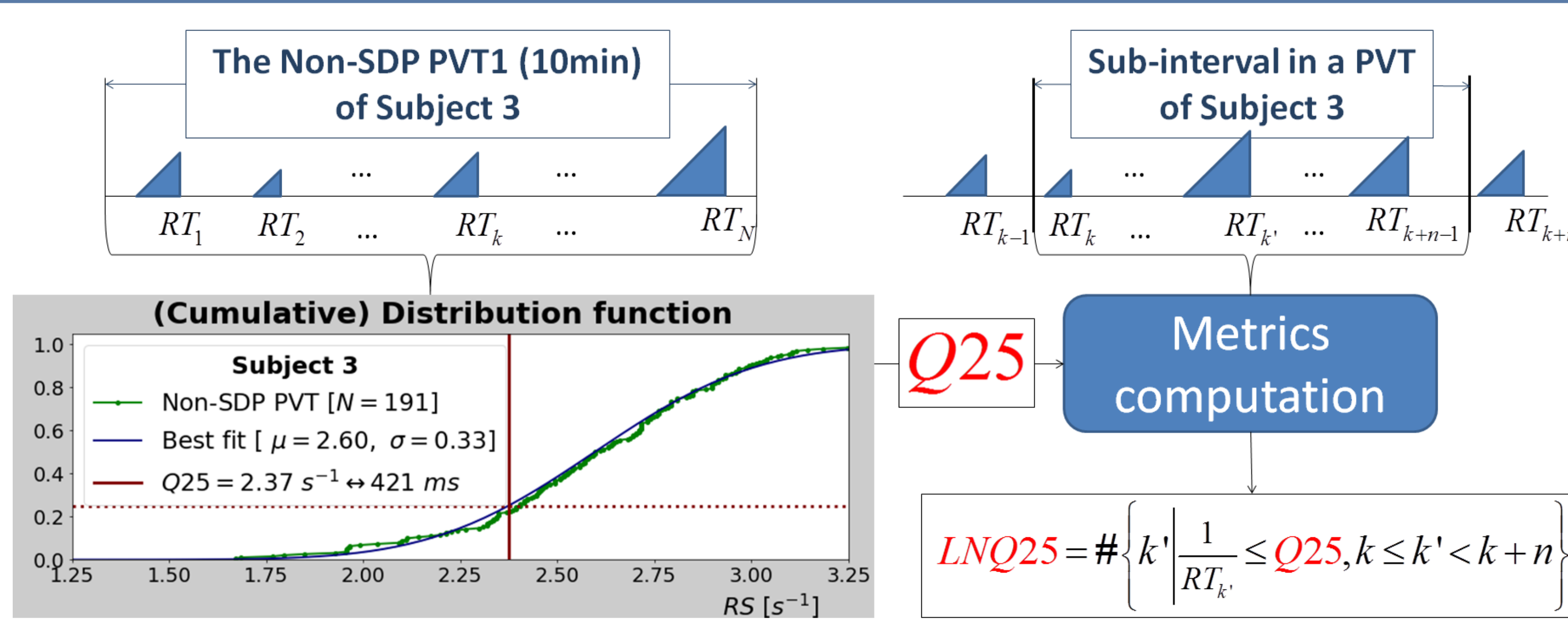

Figure 1: Computation of $\mathbf{L N Q 2 5}$ metric.
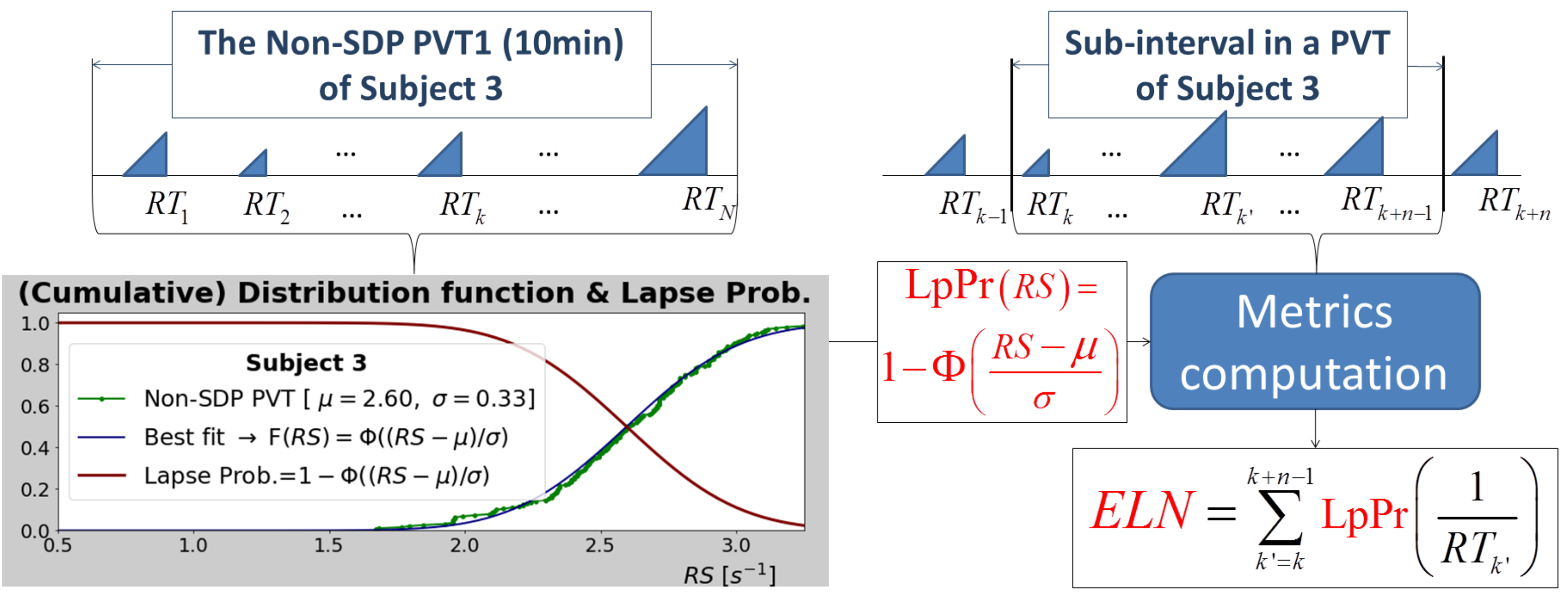

Figure 2: Computation of $\boldsymbol{E L N}$ metric.

\section{Results}

\section{Effect Size}

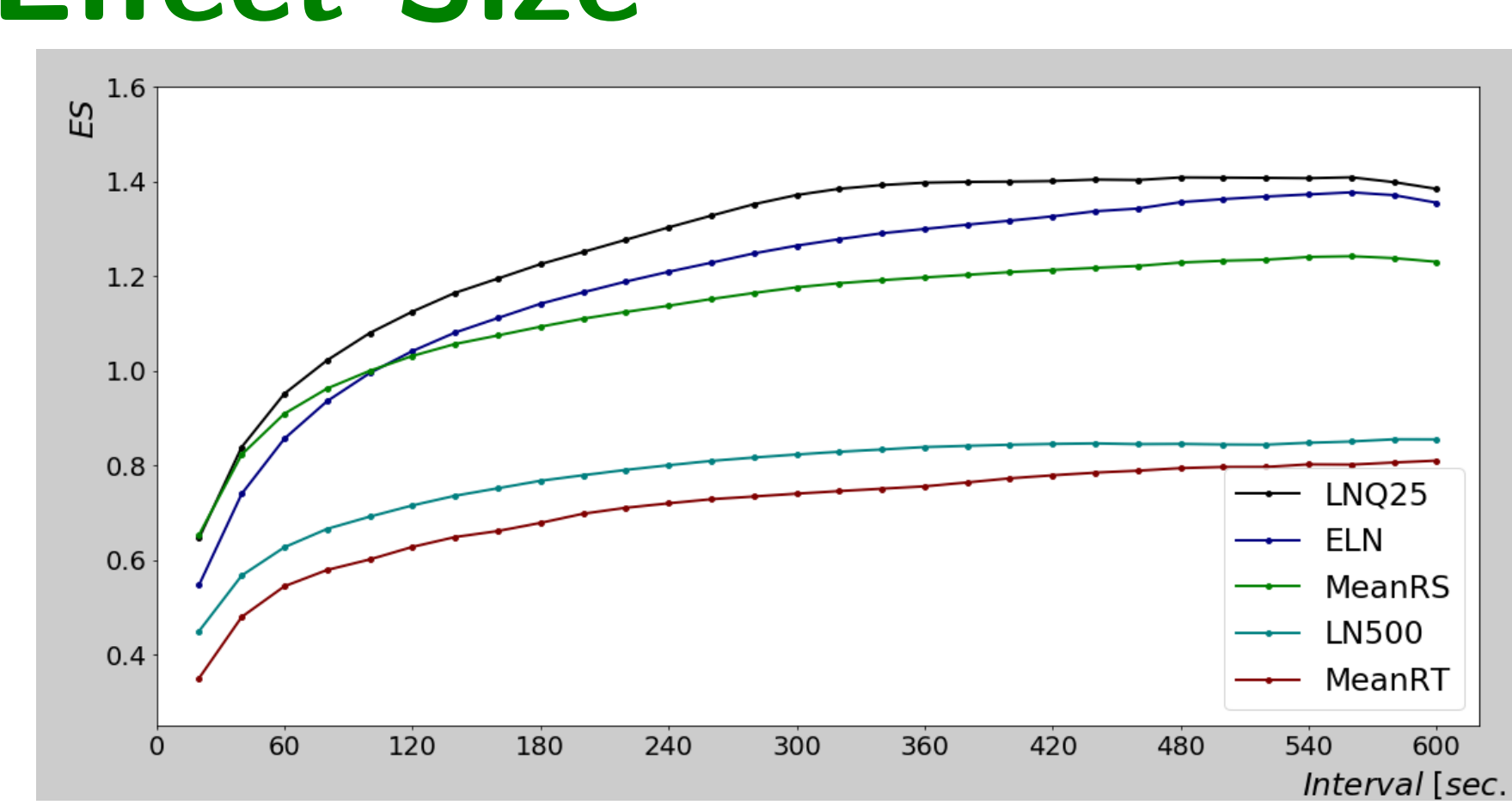

Figure 3: Effect Size of PVT metrics differences.
For a given subject, the effect of sleep deprivation is measured by the difference of PVT metrics in the SDP and Non-SDP states. The ratio of the mean by the standard deviation (for all subjects) of these differences is the "Effect Size" (ES) of sleep deprivation for the PVT metrics. [3]

\section{Subject-independent SDP/Non-SDP classification}

We consider the two distributions of the values of one PVT metric; when people are in a SDP state or not. These distributions, when computed for one subject, are often well separated by a subject-dependent threshold. The extend to which they are also clearly separated by a subject-independent threshold when computed for all subjects is one way to assess a form of independance from the subject for the PVT metrics.

\section{Evaluation of the SDP/Non-SDP classification}

We use a subject-independent threshold to class the PVT metric values (SDP/Non-SDP):

- PVT metrics values in SDP states upper (resp. lower) than the threshold are True Positive (TP) (resp. False Negative (FN)).

- PVT metrics values in Non-SDP states lower (resp. upper) than the threshold are True Negative (TN) (resp. False Positive (FP)).

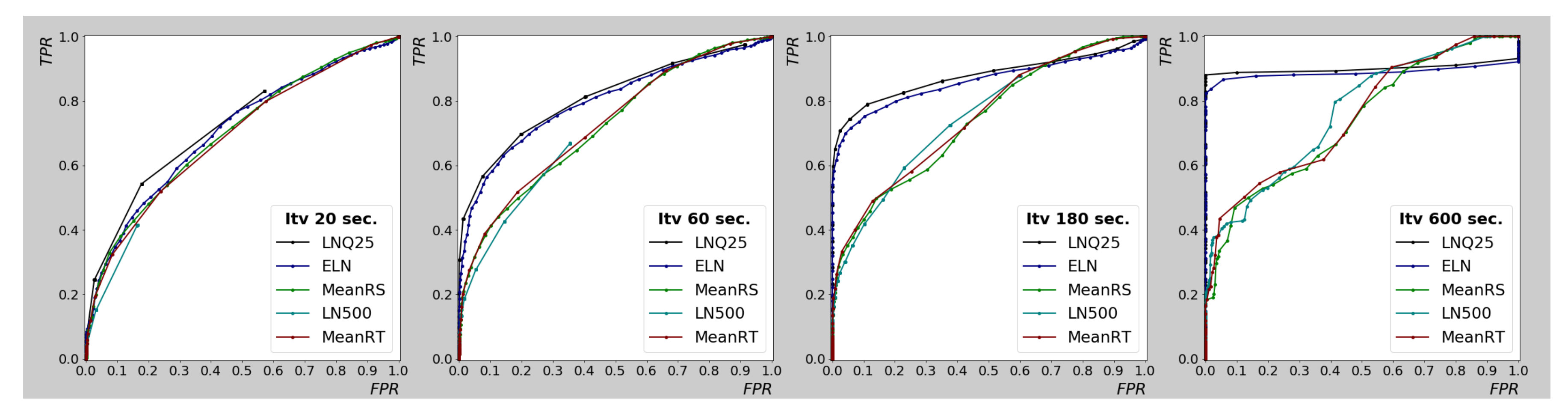

Figure 4: ROC Space for PVT Metrics.

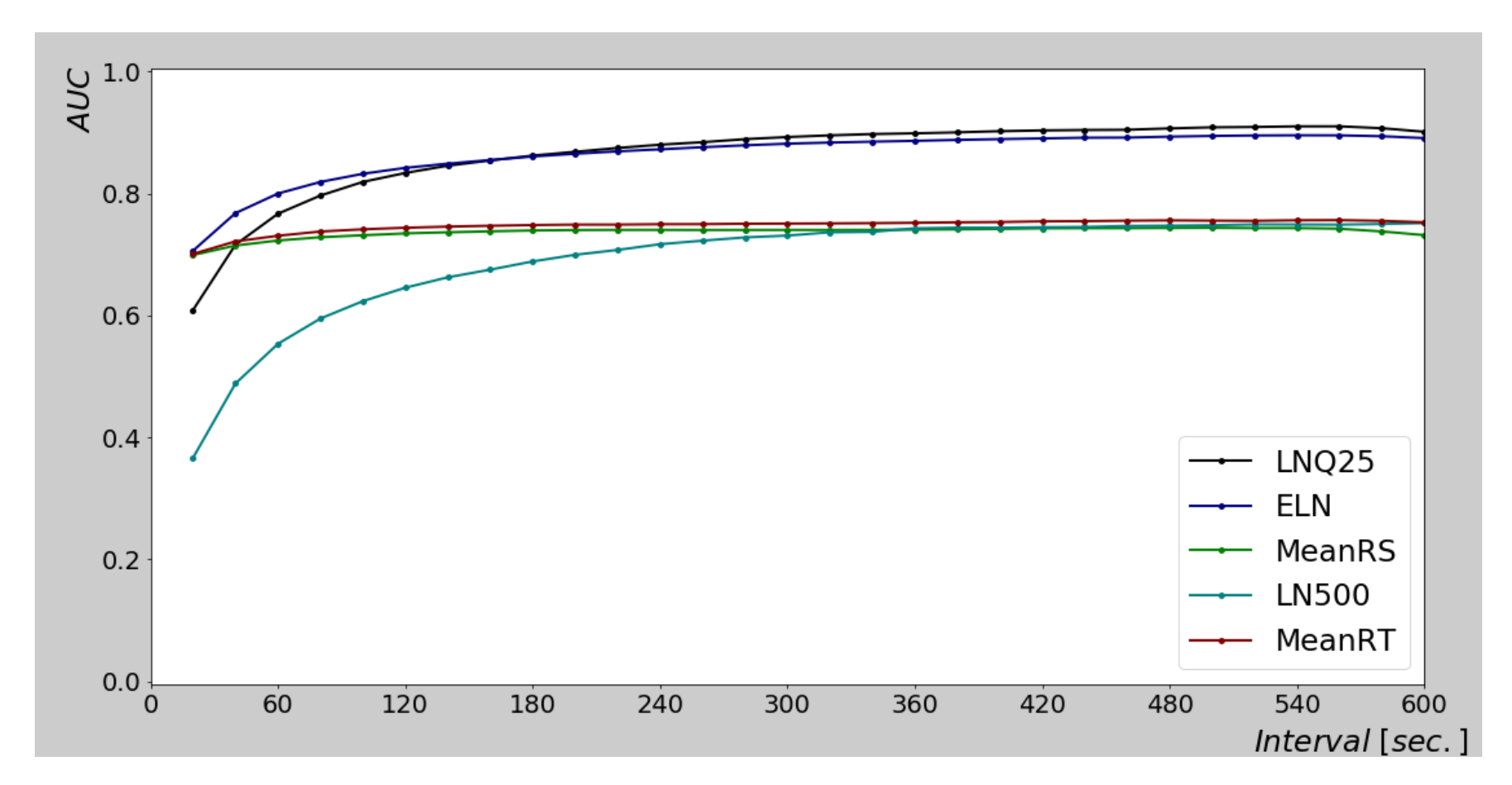

Figure 5: Area Under the ROC Curve.

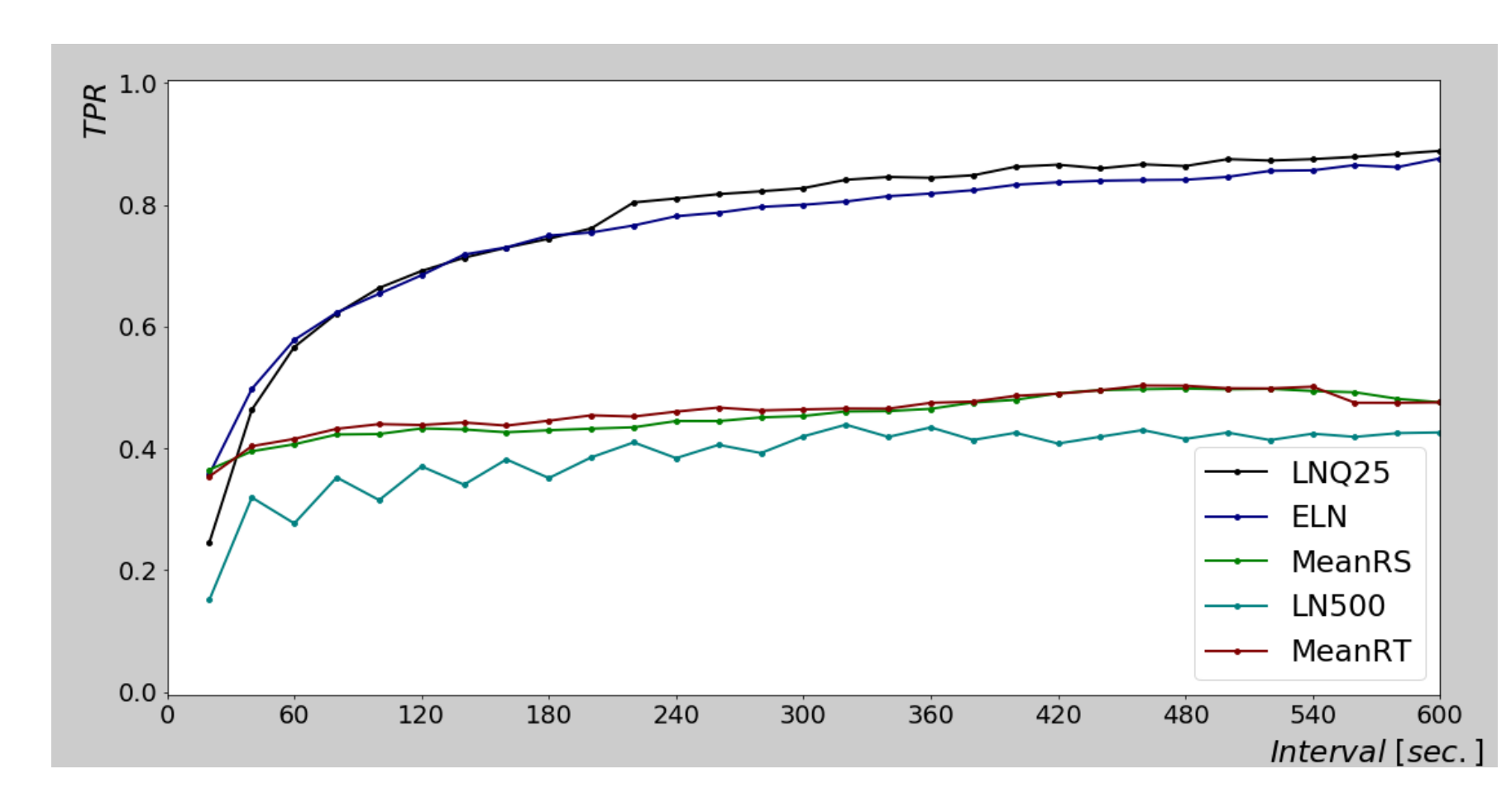

Figure 6: $\operatorname{TPR}($ with $\mathrm{FPR}=0.1$ )

\section{Conclusions}

We observed that the $L N Q 25$ and $E L N$ metrics enable a good classification of the SDP condition for time intervals greater than or equal to $3 \mathrm{~min}$, independently of the subject. And, these metrics provide also a good sensitivity to sleep deprivation. They outperform the usual metrics for both criteria. For time intervals below $3 \mathrm{~min}$, the performances first degrade progressively and then more rapidly below $1 \mathrm{~min}$.

References

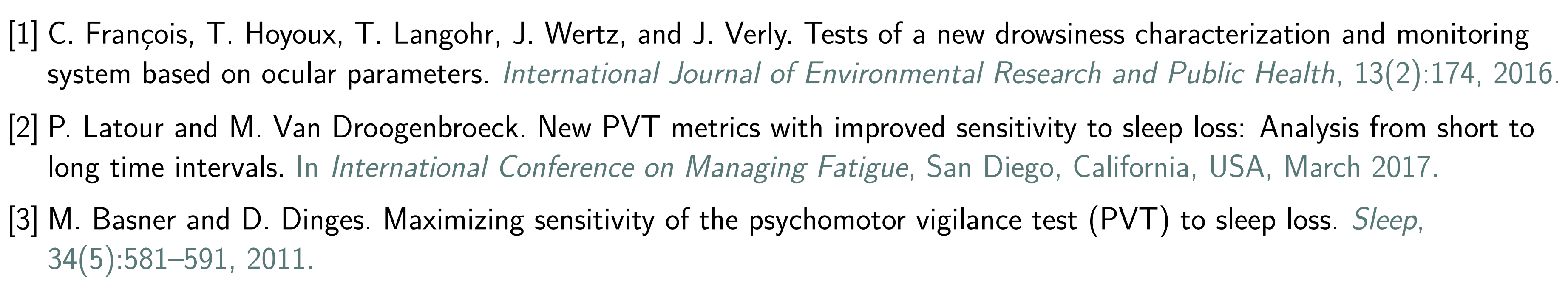

Acknowledgements

This study was supported by the University of Liège, Belgium.

The dataset has been collected with the help of the Phasya Company. 